\title{
Study of Migration of Neural Crest Cells to Adrenal Medulla by Three-Dimensional Reconstruction
}

\author{
Masako YAMAMOTO ${ }^{1)}$, Ryoko YANAI ${ }^{1)}$ and Kazuyoshi ARISHIMA ${ }^{1)}$ \\ ${ }^{1)}$ Department of Veterinary Anatomy II, Azabu University, School of Veterinary Medicine, 1-17-71 Fuchinobe, Sagamihara, Kanagawa \\ 229-8501, Japan
}

(Received 18 September 2003/Accepted 19 January 2004)

ABSTRACT. Adrenal medullary cells are derived from the neural crest. To study the formation process of the adrenal medulla in the embryonic period, we visualized chromaffin cells of rat embryos at 13 to 17 days of gestation using anti-tyrosine hydroxylase (TH) an tiserum, and created three-dimensional images from serial tissue sections. Between 13 and 15 days of gestation, TH-positive cells (chromaffin cells) migrated from a group of TH-positive cells present dorsal to the adrenal primordium via the medial cranial end of the adr enal primordium into the adrenal primordium. At or after 16 days of gestation, the adrenal capsule was formed except on the ventral aspect of the cranial end of the adrenal gland, from which TH-positive cells penetrated into the adrenal gland. The reconstructed images showed that TH-positive cells were present contiguously from the sympathetic chain ganglia through a group of TH-positive cells ventral to the adrenal gland into the adrenal cortex, and that the group of TH-positive cells ventral to the adrenal gland communicated with the preaortic ganglion present ventral and caudal to the adrenal gland. These results suggest that neural crest cells use the same pathway to migrate to the sympathetic chain ganglia dorsal to the adrenal gland, to the adrenal gland, and to the preaortic ganglion.

KEY wORDS: adrenal medulla, migration, neural crest cell.

J. Vet. Med. Sci. 66(6): 635-641, 2004

The mammalian adrenal gland consists of a cortex and a medulla. The medulla is present in the central part of the adrenal gland. Since the adrenal medullary cells have an affinity for chromium salts, they are called chromaffin cells, and synthesize and secrete the catecholamines adrenaline, noradrenaline, and enkephalin.

The adrenocortical cells are derived from the mesoderm that develops in the dorsal wall of the abdominal cavity, whereas the adrenal medullary chromaffin cells are ectoderm-derived cells migrating from the neural crest [10]. Expression of thyrosine hydroxylase $(\mathrm{TH})$, the rate-limiting enzyme of catecholamine synthesis, is first detectable in sympathetic precursors when neural crest cells begin to aggregate to form sympathetic ganglia [4, 5, 11, 12]. In adrenomedullary system, TH-immunoreactive precursor cells undergo a secondary migration from the sympathetic chain into forming adrenal gland. The precursor cells continue to express $\mathrm{TH}$-immunoreactive during migration and subsequently in the adrenal gland [3, 7, 13].

Ahonen [1] suggested that some neural crest cells continue migration from the chain ganglia to the lateral sides of the aorta, where they form the perirenal ganglia and adrenal medulla, and finally to the ventral side of the aorta to form the preaortic sympathetic tissue. However, that report only showed the several transverse sections of the fetal trunk for the a demonstration of the migration route of neural crest cells. In this study, using three-dimensional reconstructed images, we aimed to observe how TH-labeled chromaffin cells penetrate into the adrenal anlage to form the adrenal medulla and whether the migration route of $\mathrm{TH}$-positive cells from the adrenal connects the preaortic sympathetic ganglia.

\section{MATERIALS AND METHODS}

Animals: Wistar rats were kept in a semi-barrier animal facility under light-dark cycles of 12:12 hr at a temperature of $22 \pm 2{ }^{\circ} \mathrm{C}$ and humidity of $45 \%$ to $65 \%$. The animals were fed pellets (Labo MR Breeder, Nihon Nosan, Japan) and water ad libitum. Male and female rats were housed together at night. The day when the presence of sperm was confirmed on vaginal smear was defined as gestation day 0 , and an embryo on gestation day 0 was regarded as having a gestational age of 0 days.

Immunohistochemistry: At 1:00 p.m. between 13 and 17 days of gestation, mother rats were bled by decapitation, and the fetuses were taken out rapidly and, after removal of the head and chest, fixed in Bouin's solution, dehydrated in alcohol, and embedded in paraffin to prepare $5 \mu \mathrm{m}$-thick serial sections. Six fetuses from three mothers were used per each gestational day. To identify chromaffin cells under a light microscope, serial sections were stained by the ABC method using a 1:2,000 dilution of anti-TH antiserum (INCSTAR Corp., U.S.A.) as a primary antibody and biotinylated mouse IgG (Funakoshi Co., Ltd., Japan) as a secondary antibody, followed by hematoxylin counterstaining.

Three-dimensional reconstructed method: Images of all the serial stained sections were digitized by a KODAK DC40 digital camera (Kodak Japan Ltd.) attached to an OLYMPUS BX50 light microscope (Olympus, Japan), and input into Photo Enhancer image-processing software (Kodak Japan Ltd.) in a Power Macintosh (Apple Japan, Inc.) to make slides. These slides were input into Adobe Photoshop 5.0 image-editing software (Adobe Systems, The Netherlands), and chiefly edited as follows: (1) all data were standardized to $800 \times 800$ pixels, (2) directions as well as 
positions were fitted to one another, and unnecessary portions were deleted, (3) color images were turned into gray scale images to input them into volume-rendering software, (4) densities were equalized, and (5) since no capsule was formed around the adrenal primordium between 13 and 15 days of gestation, the densities of TH-positive cells in the adrenal primordium were reduced, and, at 16 and 17 days of gestation, the densities of TH-positive cells were raised to visualize them. The data thus edited were input into Voxel/ View volume-rendering software (Vital Image Inc., U.S.A.) to generate three-dimensional images. To prevent the shift of $\mathrm{z}$-axis, the position of the notochord and the inner space of spinal cord were used to fit $\mathrm{z}$-coordination.

\section{RESULTS}

Figure 1A shows the three-dimensional reconstructed images at 17 days of gestation observed from the ventral, right oblique, cranial direction. The spinal cord, dorsal artery, and liver as well as the cross section of the kidney were observed. When the image in Fig. 1A was processed to enhance the contour of the adrenal gland and the TH-positive cells, the TH-positive cells (black) became visible outside the contour (gray) of the adrenal gland and in the adrenal cortex (Fig. 1B). Since the adrenal capsule was formed at 16 and 17 days of gestation, and the boundary between the adrenal gland and the surrounding tissue became well defined, we were able to delete the structures other than the adrenal gland and TH-positive cells (Fig. 1C).

Thirteen days of gestation: The adrenal cortical primordium consists of a small group of cells located in the dorsal wall on both sides of the dorsal artery, and was poorly demarcated from the surrounding tissue (Figs. 2A, 2B). THpositive cells penetrating from behind the adrenocortical primordium into the adrenal gland were observed (Fig. 2B). The three-dimensional reconstructed images generated from these tissue sections showed that a small group of TH-positive cells representing an apparent sympathetic ganglion was present behind the TH-positive cells (gray dots) that had penetrated into the adrenal gland (Fig. 3A).

Fourteen days of gestation: The adrenal cortical primordium was slightly enlarged, and TH-positive cells were present in a group dorsal to the adrenal gland, in the adrenal primordium, and in a line connecting the adrenal primordium and the dorsal group of TH-positive cells (Fig. 2C). The three-dimensional reconstructed images showed that a group of TH-positive cells representing apparent the sympathetic chain ganglia was present in the long axis direction behind the intraadrenal TH-positive cells (Fig. 3B).

Fifteen days of gestation: The adrenal primordium enlarged rapidly, and TH-positive cells were scattered throughout the adrenal primordium, but tended to be abundant in the dorsal part of the adrenal gland (Fig. 2D). THpositive cells formed a group toward the center of the dorsal part of the adrenal gland, and were present also in a line connecting this group and the medial end of the adrenal primordium. Capsule covering the adrenal primordium was
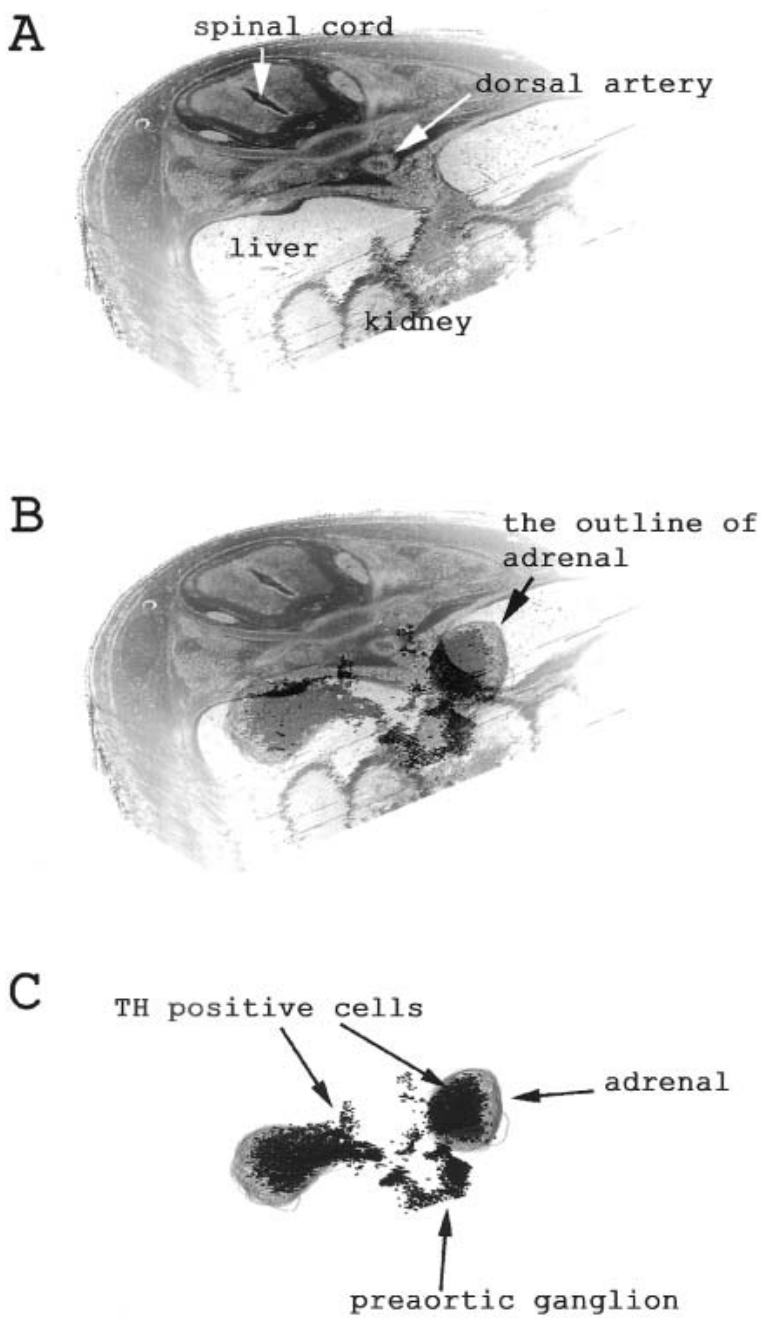

Fig. 1. Three-dimensional reconstructed images at 17 days of gestation. A: Observation from the ventral, right oblique, cranial direction. B: Image obtained by processing the image in A to enhance the contour of the adrenal gland and the TH-positive cells. C: Image obtained by deleting the structures other than the adrenal gland and TH-positive cells.

formed yet. Three-dimensional images showed that THpositive cells aggregated cranial to caudal to the adrenal gland (Fig. 3C). Dorsal to the TH-positive cells present in the adrenal gland, TH-positive cells formed the sympathetic chain ganglia (Fig. 3C, arrow). Caudal to the intraadrenal group of TH-positive cells, a group of cells was present.

Sixteen days of gestation: The adrenal gland had dramatically grown (Fig. 4A). Head-to-tail sequential observation of serial sections showed that, at 16 days of gestation, a small group of TH-positive cells was recognizable as a sympathetic ganglion surrounded by a perineurium, and some of the ganglion cells were TH-positive. In the vicinity of the ventral medial part of the adrenal gland, a group of TH-positive cells was present, and, between these TH-positive cells and the intraadrenal TH-positive cells, TH-positive cells 

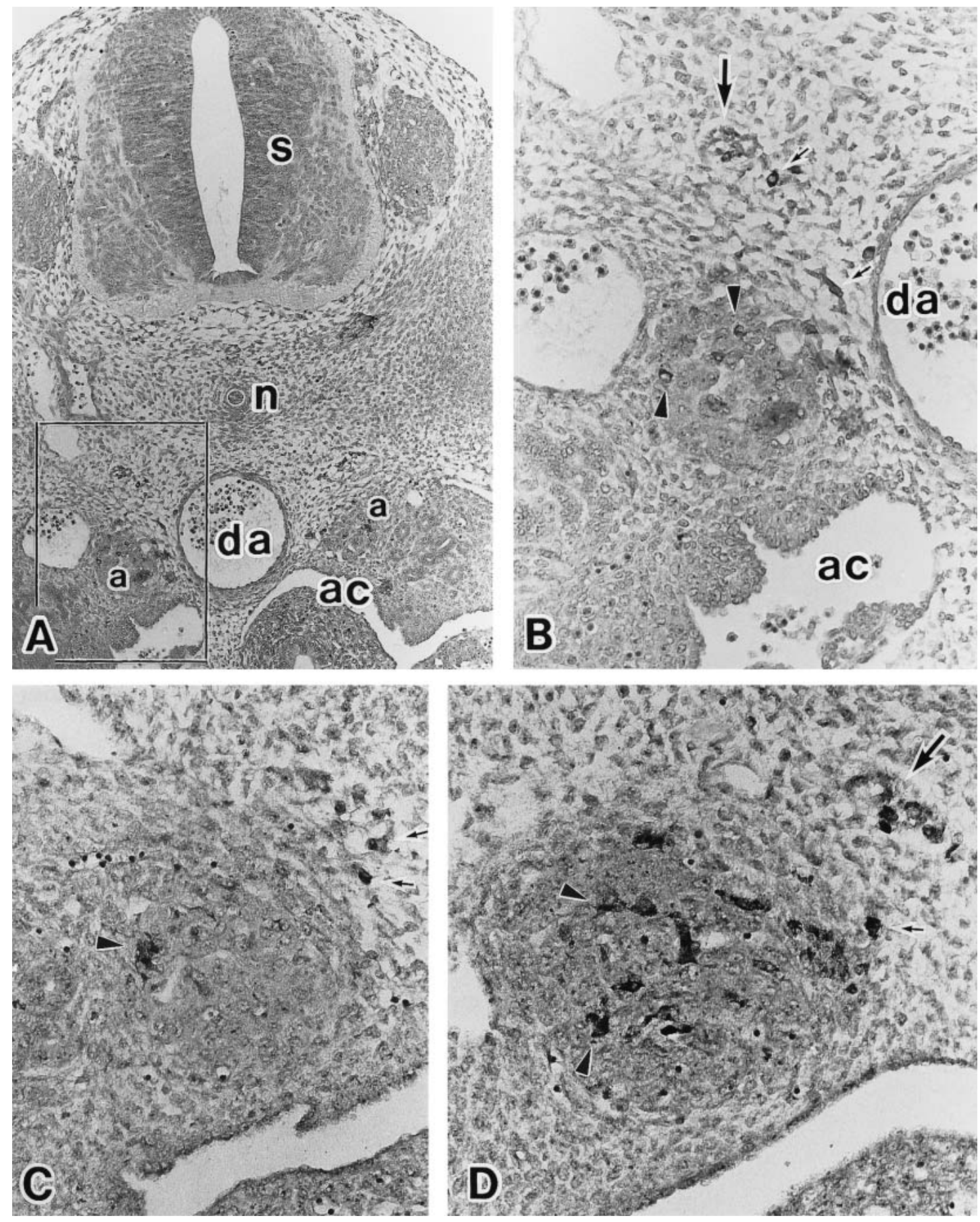

Fig. 2. Photomicrograph of the cross section of an embryo (dorsal at top). A: Photomicrograph at 13 days of gestation. The adrenal primordia (a) were present in the dorsal wall on both sides of the dorsal aorta (da). s; spinal cord, n; notochord, ac; abdominal cavity. $\times 84$. B: Enlarged photograph of the encircled part of A. Arrowheads; TH-positive cells in the adrenal primordium, large arrow;mass of TH-positive cells, small arrows; TH-positive cells in the line connecting the adrenal primordium and the mass of TH-positive cells. $\times 212$. C: Photomicrograph of the adrenal primordium at 14 days of gestation. Arrowhead; TH-positive cells in the adrenal primordium, arrows; TH-positive cells in the line connecting the adrenal primordium and the mass of TH-positive cells. $\times 256$. D: Photomicrograph of the adrenal primordium at 15 days of gestation. Arrowheads; TH-positive cells in the adrenal primordium, large arrow; the sympathetic chain ganglion, small arrow; THpositive cells in the line connecting the adrenal primordium and the sympathetic chain ganglion. $\times 256$. 


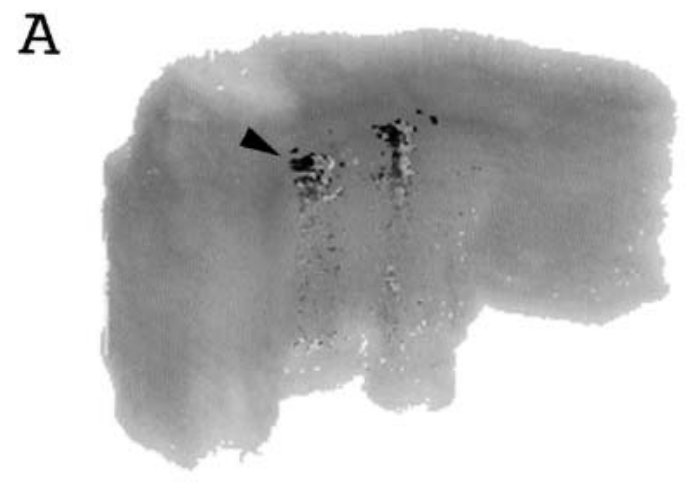

B
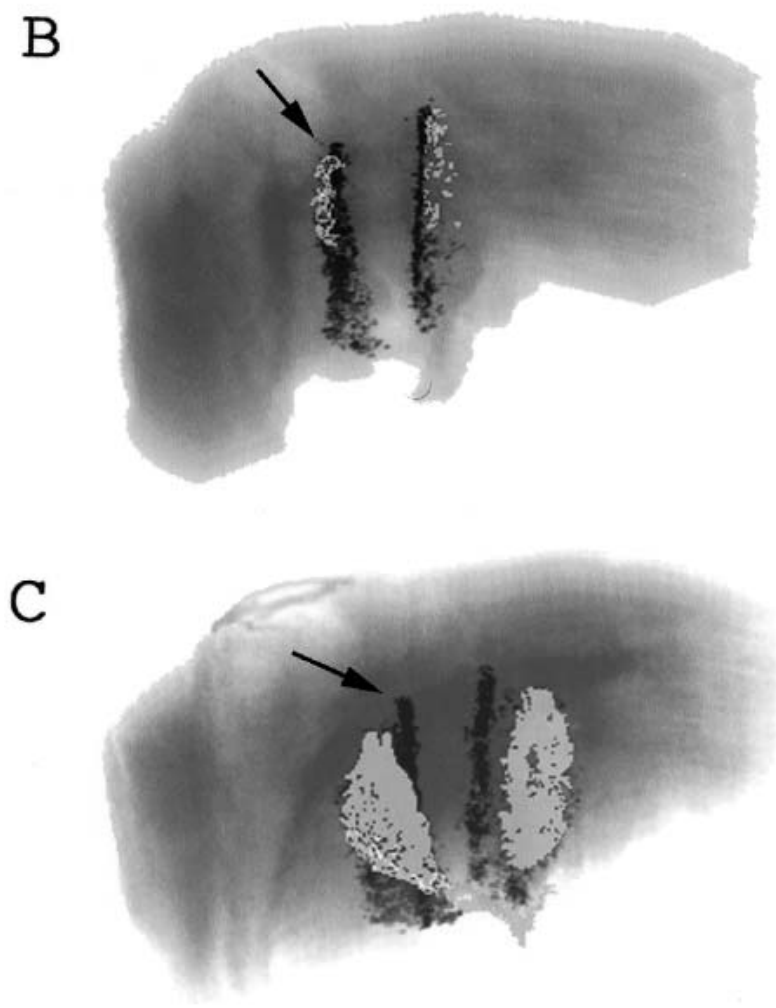

Fig. 3. Three-dimensional reconstructed image of an embryo trunk viewed from the upper right oblique direction, processed to enhance only the TH-positive cells. Gray dots; TH-positive cells in the adrenal primordium, black dots: Cells other than the TH-positive cells, arrowhead; ganglion, arrow; the sympathetic chain ganglion. A: 13 days of gestation. B: 14 days of gestation. C: 15 days of gestation.

were present. Unlike around the sympathetic ganglion, no perineurium-like structures were observed around the group of TH-positive cells in the vicinity of the ventral medial part of the adrenal gland. The more caudal the tissue section, the more nerve fibers were seen to connect the sympathetic ganglion and the group of TH-positive cells. As the cranial end of the kidney began to be visible ventral to the adrenal cortex, a distance separated the group of TH-positive cells and the adrenal gland, resulting in loss of continuity with the TH-positive cells in the adrenal cortex. More caudally, the left and right groups of TH-positive cells joined to form a group, that is, the preaortic ganglion. A capsule began to form around the adrenal gland (Fig. 4A), making the contour of the adrenal gland well defined. However, no capsule was formed in the ventral medial part of the adrenal gland, and blood vessels penetrated into the adrenal cortex from this site.

Three-dimensional images observed from the right upper oblique and ventral median directions demonstrated that the TH-positive cells in and ventral to the adrenal cortex were contiguous with the extraaderenal group of $\mathrm{TH}$-positive cells (Figs. 5A, 5B). We found that two extraaderenal groups of TH-positive cells were present: one connecting with the sympathetic chain ganglia dorsal to the adrenal gland, and the other contiguous with the preaortic ganglion ventral and caudal to the adrenal gland.

Seventeen days of gestation: The cross section at 17 days of gestation was larger than that at 16 days of gestation. As at 16 days of gestation, TH-positive cells were present in the adrenal cortex, the sympathetic chain ganglia (Fig. 4B), and preaortic ganglion. Also, a large group of TH-positive cells was present in the vicinity of the medial part of the adrenal gland. As at 16 days of gestation, no capsule was formed in the ventral medial part of the adrenal gland. TH-positive cells were present also in the sympathetic ganglion caudal to the adrenal gland.

The three-dimensional reconstructed images viewed from the right upper oblique and ventral median directions clearly showed that the adrenal gland was increased in volume, and that a large group of TH-positive cells around the adrenal gland in the vicinity of the ventral medial part of the adrenal gland was contiguous with the TH-positive cells in the adrenal cortex (Figs. 5C, 5D). However, the continuity of the extraaderenal group of TH-positive cells with the sympathetic chain ganglia was scarcer than that at 16 days of gestation, and the line of TH-positive cells connecting with the caudally located preaortic ganglion was also weaker (Figs. $5 \mathrm{C}, 5 \mathrm{D})$. As at 17 days of gestation, TH-positive cells were also seen in the preaortic ganglion, which was outside the site of the tissue section used for stereo imaging and is therefore not shown in the reconstructed image.

\section{DISCUSSION}

According to Kent and Row [8], neural crest-derived chromaffin cells are present between the dorsal root ganglia and the medial pole of the adrenocortical primordium in 12day-old rat embryos, and are seen in the central part of the adrenal cortex singly or in small groups at 13 days of gestation. Chromaffin cells themselves aggregating in the center of the adrenal medulla by 16.5 days (corresponding to 16 days in this study) of gestation are said to become enlarged. Also, the group of cells grows and assumes the morphology of the adrenal medulla. Neural crest cells have been reported to migrate into sympathetic ganglia and the adrenal 

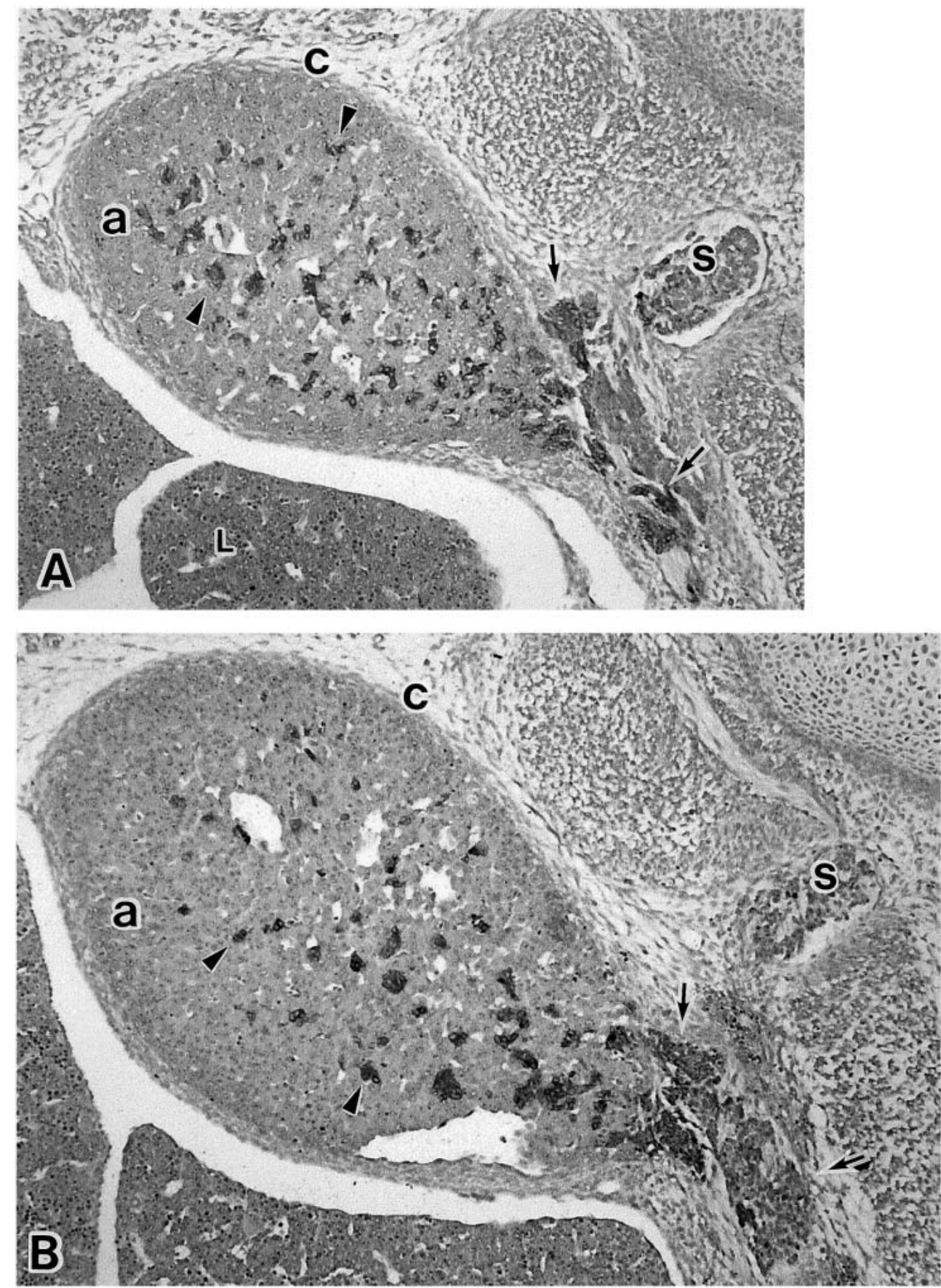

Fig. 4. Photomicrographs of the cross sections of a 16-day-old (A) and a 17-day-old (B) embryo. a;adrenal gland, arrows;mass of extra-adrenal TH-positive cells, arrowheads; TH-positive cells in adrenal gland, c; adrenal capsule, L; liver, s; the sympathetic chain ganglion, $\times 200$.

cortex, and almost completely convert to chromaffin cells by 19 days of gestation, forming the adrenal medulla [2]. Since TH used in this study as an indicator of chromaffin cells (adrenal medullary cells) is an enzyme involved in the early stage of the synthetic pathway of adrenaline and noradrenaline, the TH-positive cells observed in this study may include not only chromaffin cells but also sympathetic ganglion cells and SIF cells [6] with catecholamine-containing granules. However, it has been suggested that, in anin vitro experimental system, neural crest-derived adrenergic precursors convert to chromaffin cells under the action of steroid hormone when they penetrate into the adrenal gland via 

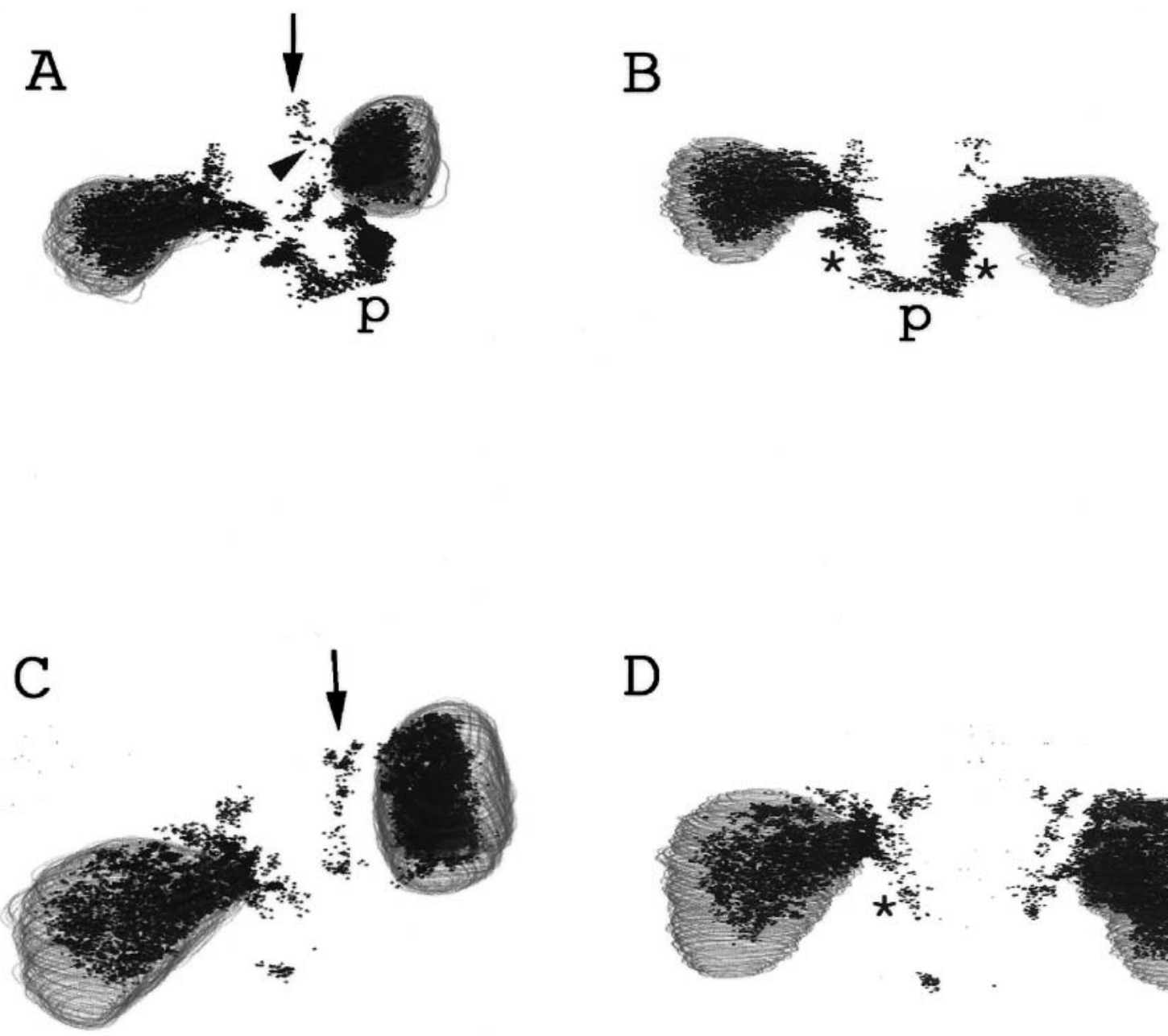

Fig. 5. Reconstructed images processed to enhance only the TH-positive cells and the contour of the adrenal gland. A \& B: 16 days of ges tation. C \& D; 17 days of gestation. A \& C: Semilateral view. B \& D: Ventral view. Arrows; the sympathetic chain ganglion, arrowheads; the line of TH-positive cells from the sympathetic chain ganglion to the adrenal, asterisks; the line of TH-positive cell s from the adrenal to the preaortic ganglion, p; preaortic ganglion.

sympathetic ganglia [2]; therefore, the TH-positive cells observed in the adrenal gland in this study presumably represent chromaffin cells.

Reconstructed images showed that the apparent site of penetration of the adrenal primordium by TH-positive cells was at the dorsal medial part of the adrenal cortical primordium at 13 to 15 days of gestation, but it had moved to the ventral medial part at 16 and 17 days. Kent and Rowe [2] reported that the apparent site of penetration of the adrenal cortex by TH-positive cells had no capsule, and that chromaffin cells present in this break in the adrenal capsule of 18-day-old rat embryos would extend into the cortex. This study also showed that a capsule was first formed around the adrenal gland at 16 days of gestation, and that TH-positive cells penetrated into the adrenal gland from the ventral medial part of the adrenal gland where the capsule was undeveloped. In other words, the break in the capsule at the ventral medial part of the adrenal gland is the doorway to blood vessels and nerves including TH-positive cells, apparently corresponding to the hilum of other organs.

At 13 days of gestation, only a small group of TH-positive cells was present dorsal to the intraadrenal $\mathrm{TH}$-positive cells. However, at 14 days of gestation, a long, narrow group of TH-positive cells was seen extending in the direction of the long axis of the embryo, indicating that the lumbar sympathetic ganglia are formed in rat embryos at 14 days of gestation. At 15 days of gestation, the development of the sympathetic chain ganglia was more pronounced. The reconstructed images at 16 and 17 days of gestation suggest that TH-positive cells that have migrated from the sympathetic chain ganglia into the adrenal gland pass the vicinity of the ventral medial part of the adrenal gland, and migrate toward the preaortic ganglion. It was previously thought that when neural crest-derived cells migrated via the sympathetic chain ganglia into the adrenal gland or preaortic ganglion, they took different pathways to the respective des- 
tinations at the time of passage through the ganglia of the sympathetic trunk [9]. However, the reconstructed images in this study showed that the pathway of neural crestderived cells from the ganglia of the sympathetic trunk to the adrenal medulla overlapped with the pathway to the preaortic ganglion. We speculate that neural crest cells use the same pathway to migrate to the ganglia of the sympathetic trunk, to the adrenal gland, and to the preaortic ganglion, this conclusion is good agreement of the suggestion by Ahoren [1]. The finding that the line of TH-positive cells toward the adrenal gland almost disappeared at 17 days of gestation in the reconstructed image suggests that the migration of neural crest-derived cells to the adrenal gland is complete by the 17 days of gestation, after which the formation of the adrenal medulla is completed by cell division in the adrenal gland.

\section{REFERENCES}

1. Ahnen, M. 1991. Neurofilament immunoreactivity and acethylcholinesterase activity in the developing sympathetic tissue of the rat. Histochemistry 96: 467-478.

2. Anderson, D.J. and Axel, R. 1986. A bipotential neuroendocrine precursor whose choice of cell fate is determined by NGF and glcocorticoids. Cell 47: 1079-1090.

3. Bohn, M.C., Goldstein, M. and Black, I.B. 1981. Role of glucocorticoids in expression of the adrenergic phenotype in rat embryonic adrenal gland. Dev. Biol. 82: 1-10.

4. Cochard, P., Goldstein, M. and Black, I. 1979. Initial development of the noradrenergic phenotype in automatic neuroblasts of the rat embryo in vivo. Dev. Biol. 71: 100-114.

5. DeChamplain, J., Malmfos, T., Olson L. and Sachs, C. 1970.
Ontogenesis of peripheral adrenergic neurons in the rat: preand postnatal observations. Acta Physiol. Scand. 80: 276-288.

6. Doupe, A.J., Patterson, P.H. and Landis, S.C. 1985. Environmental influence in the development of neural crest derivatives: glucocorticoids, growth factors and chromaffin cell plasticity. J. Neurosci. 5: 2143-2160.

7. Henion, P.D. and Landis, S.C. 1990. Asynchronous appearance and topographic segregation of neuropeptide-containing cells in the developing rat adrenal medulla. J. Neurosci. 10: 2886-2896.

8. Kent, C. and Rowe, H.L. 1992. The immunolocalization of ubiquitin carboxyl-terminal hydrolase (PGP9.5) in developing paraneurons in the rat. Dev.Brain Res. 68: 241-246.

9. Laesen, W.J. 1997. Human Embryology, 2nd ed., Churchill Livingstone Inc., New York.

10. Pankrats, D.S. 1931. The development of the suprarenel gland in the albio rat. Anat. Rec. 49: 31-39.

11. Rothman, P.R., Specht, L.A., Gershon, M.D., Joh, T.H., Teitelman, G., Pickel, V.M. and Reis, D.J. 1980. Catecholamine biosynthetic enzymes are expressed in replicating cells in the peripheral but not the central nervous systems. Proc. Natl. Acad. Sci. U.S.A. 77: 6221-6225.

12. Teitelman, G., Baker, H., Joh, T.H. and Reis, D.J. 1979. Appearance of catecholamine-synthesizing enzymes during development of the rat sympathetic nervous system: possible role of tissue environment. Proc. Natl. Acad. Sci. U.S.A. 76: 509-513.

13. Verhofstad, A.A.J., Hökfelt, T., Goldstein, M., Seinbusch, H.W.M. and Joosten, H.W.J. 1979. Appearance of thyrosine hydroxylase, aromatic amino acid decarboxylase, dopamine Bhydroxylase and phenylethanolamine N-methyltrasferase during the ontogenesis of the adrenal medulla. Cell Tissue Res. 200: $1-13$. 